\title{
Respiration-Guided Analysis of Pulse and Heart Rate Variabilities for Acute Emotional Stress Assessment
}

\author{
Jorge Mario Garzón-Rey ${ }^{1,2}$, Jesús Lázaro ${ }^{3,4}$, Javier Milagro ${ }^{2,5}$, Eduardo Gil ${ }^{2,5}$, Jordi Aguiló1,2, \\ Raquel Bailón ${ }^{2,5}$ \\ ${ }^{1}$ Microelectronics and Electronic Systems Department. Autonomous University of Barcelona, \\ Bellaterra, Spain \\ ${ }^{2}$ Centro de Investigación Biomédica en Red (CIBER-BBN), Madrid, Spain \\ ${ }^{3}$ Department of Electrical Engineering (ESAT), STADIUS, KU Leuven, Leuven, Belgium \\ ${ }^{4}$ IMEC, Leuven, Belgium \\ ${ }^{5}$ BSICoS Group, Aragón Institute for Engineering Research (I3A), IIS Aragón, University of \\ Zaragoza, Zaragoza, Spain
}

\begin{abstract}
Acute Emotional Stress may be assessed through the changes on the autonomic nervous system. Pulse Rate Variability $(P R V)$ and Heart Rate Variability $(H R V)$ are well known and widely used as markers of autonomic nervous system. Pulse photoplethysmographic and electrocardiographic signals were synchronously recorded during relax and stress stages from 80 young healthy students during an ad hoc experiment. To evaluate the agreement among $H R V$ and $P R V$, frequency indices were computed over 1-min-length running window on the classical spectral bands [LF:0.04-0.15Hz, HF:0.15-0.4Hz], extended HF band [0.15Hz-half mean Heart Rate], HF band centered at respiratory frequency with a bandwidth of 0.1 $H z$. Between relax and highest stress stage most of the $H R V$ indices show significant changes but most of the PRV indices do not. Most of the indices showed significant differences between PRV and HRV on relax stages. In contrasts, during stress stages they do not show significant differences. These results suggest that pulse arrival time variability is higher during relax.
\end{abstract}

\section{Introduction}

As response of a stress stimulus the autonomic nervous system generates an inhibition of the parasympathetic nervous system and an activation of the sympathetic nervous system [1].

Both systems act in the opposite way generating activity in the adrenal medulla that is reflected in the increase of the heart rate, the blood pressure, the bronchodilation, the sweating, the metabolic activity and the concentration of glucose in the blood[2].

Pulse Rate Variability (PRV) and Heart Rate Variability (HRV) are widely used tools for non-invasive autonomic nervous system assessment. Although some differences in indices extracted from HRV and PRV exist, PRV could be used as a surrogate of HRV during the tilt table test[3].

The ES3 Project [4], attempting to develop a continuously and a non-invasively measurement of acute emotional stress in young people, in relation to assess stress has analyzed HRV and respiratory frequency pointing out the importance of taking into account respiratory information in HRV analysis [5].

Due to the fact that HRV and PRV may provide different but complementary information, this paper presents a reliability comparison between HRV and PRV including respiratory frequency information for acute emotional stress assessment.

\section{Materials and Methods}

\subsection{Database}

Respiratory signal, pulse photoplethysmographic (PPG) and electrocardiographic (ECG) signals were synchronously recorded during relax and different stress states from 80 young healthy students at Autonomous University of Barcelona (Spain) and University of Zaragoza (Spain). PPG and respiratory signal were sampled at $250 \mathrm{~Hz}$ and ECG was sampled at $1 \mathrm{kHz}$ by using ABP-10 module of the Medicom device (Medicom MTD Ltd.). Relax and stress states were achieved by using the experimental protocol described in [5]. The following stages of the protocol were analyzed: prerelaxing stage before relax (PRB), deep relax $(\mathrm{R})$, prerelaxing stage before stress (PSB), story 
telling stage (ST), where subjects were listening to stories that they had to remember, stress anticipation (SA), where subjects were waiting for their evaluation, and video exposition stage (V), where a video showing the subject's performance was displayed in front of an audience. Other stages of the experimental protocol were excluded because they required the subject to speak.

\subsection{Respiratory frequency estimation}

Based on respiratory frequency estimation described in [5], the respiratory signal was filtered with a pass-band filter (cutoff frequencies of $0.03 \mathrm{~Hz}$ and $0.9 \mathrm{~Hz}$ ) and downsampled from $250 \mathrm{~Hz}$ to $4 \mathrm{~Hz}$. For each stage respiratory frequency $\left(F_{\mathrm{R}}(k)\right)$ was estimated on 1-min-length windows as position of the maximum of the power spectrum density $\left(S_{k}(f)\right)$ of the respiratory signal. $F_{\mathrm{R}}(k)$ was considered only if the peakness $\left(P_{k}\right)$ of $S_{k}(f)$ is higher than $65 \%$. $P_{k}$ was defined as:

$$
P_{k}=\frac{\int_{F_{\mathrm{R}}(k)-0.1}^{F_{\mathrm{R}}(k)+0.1} S_{k}(f) d f}{\int_{0.08}^{0.8} S_{k}(f) d f} \cdot 100
$$

\subsection{Electrocardiography}

Heart beats were detected from the ECG signal using an algorithm based on the discrete wavelet transform [6]. In addition, an instantaneous Heart rate signal (HR) was obtained from the beat occurrence time series using an algorithm based on the integral pulse frequency modulation model, which takes into account the presence of ectopic beats [7].

\subsection{Pulse photoplethysmography}

PPG signal was filtered using a low pass FIR filter with a cut-off frequency of $35 \mathrm{~Hz}$ (order 50). Then a high pass FIR filter with a cut-off frequency of $0.3 \mathrm{~Hz}$ (order 5000) was applied to the signal. PPG artifacts were suppressed through a Hjorth-parameter-based PPG artifact detector described in [8]. Pulses were detected from the PPG signal on those time slots without artifacts by an algorithm based on [9]. Finally, an instantaneous Pulse rate signal (PR) was obtained using an algorithm based on the integral pulse frequency modulation model, as with the HR signal [7].

\subsection{Pulse and heart rate variabilities in- dices}

The following PRV and HRV frequency indices were computed over the same 1-min-length running window from synchronous PR signal and HR signal: Power on Low frequency $\left(P_{\mathrm{LF}}\right)$ and High frequency $\left(P_{\mathrm{HF}}\right)$ classical spectral bands [LF:0.04-0.15Hz, HF:0.15-0.4Hz]. Power in extended high frequency $(\mathrm{HF})$ band $\left(P_{\mathrm{HFex}}\right)[0.15 \mathrm{~Hz}$-half mean Heart Rate]. Power in HF band centered at respiratory frequency $\left(P_{\mathrm{HFr}}\right)\left[F_{\mathrm{R}}(k)-0.05, F_{\mathrm{R}}(k)+0.05 \mathrm{~Hz}\right] . P_{\mathrm{LF}}$ and $P_{\mathrm{HF}}$ normalized by the sum of $P_{\mathrm{LF}}$ and $P_{\mathrm{HF}}\left(P_{\mathrm{LFn}}\right.$ and $\left.P_{\mathrm{HFn}}\right)$. $P_{\mathrm{LF}}$ and $P_{\mathrm{HFex}}$ normalized by the sum of $P_{\mathrm{LF}}$ and $P_{\mathrm{HFex}}\left(P_{\mathrm{LFexn}}\right.$ and $\left.P_{\mathrm{HFexn}}\right) . P_{\mathrm{LF}}$ and $P_{\mathrm{HFr}}$ normalized by the sum of $P_{\mathrm{LF}}$ and $P_{\mathrm{HFr}}\left(P_{\mathrm{LFrn}}\right.$ and $\left.P_{\mathrm{HFrn}}\right)$. Ratio between $P_{\mathrm{LF}}$ and $P_{\mathrm{HF}}(L F / H F)$. Ratio between $P_{\mathrm{LF}}$ and $P_{\mathrm{HFex}}(L F / H F e x)$. Ratio between $P_{\mathrm{LF}}$ and $P_{\mathrm{HFr}}(L F / H F r)$. Every time window where $F_{\mathrm{R}}(k)$ falls within the LF band were excluded as in [5].

Indices from PRV were compared with those from HRV using differences of least squares means (DLSM) of fixed effects from linear mixed models with the subject as a random effect. For Non-Normal distributed indices the BoxCox were used.

\section{Results}

Percentage change between prerelaxing stage before relax (PRB) and prerelaxing stage before stress (PRS) for HRV and PRV indices are show in Table 1. HRV and PRV indices related to $\mathrm{HF}$ band centered at $F_{\mathrm{R}}$ and related to $\mathrm{HF}$ extended band are not significantly different between PRB and PRS, except for $P_{\mathrm{HFex}}$ and $P_{\mathrm{HFexn}}$ in HRV. For classic band only $P_{\mathrm{LF}}$ is not different between PRB and PRS.

Table 1. Percentage change between prerelaxing stage before relax (PRB) and prerelaxing stage before stress (PRS) for HRV and PRV indices. Values in [\%] *p-value $<0.05$

\begin{tabular}{lrr}
\hline \hline & \multicolumn{2}{c}{ PRB vs PRS } \\
\cline { 2 - 3 } & \multicolumn{1}{c}{$\mathrm{HRV}$} & \multicolumn{1}{c}{$\mathrm{PRV}$} \\
\hline$P_{\mathrm{HF}}$ & $-14.43^{*}$ & $-12.61^{*}$ \\
$P_{\mathrm{HFr}}$ & -6.60 & -4.84 \\
$P_{\mathrm{HFex}}$ & $-10.77 *$ & -9.11 \\
$P_{\mathrm{HFn}}$ & $-4.34^{*}$ & $-4.00^{*}$ \\
$P_{\mathrm{HFrn}}$ & -2.26 & -1.78 \\
$P_{\mathrm{HFexn}}$ & $-3.72^{*}$ & -3.36 \\
$L F / H F$ & $14.90^{*}$ & $13.75^{*}$ \\
$L F / H F r$ & 8.82 & 7.53 \\
$L F / H F e x$ & 12.08 & 10.97 \\
$P_{\mathrm{LF}}$ & 3.01 & 3.22 \\
$P_{\mathrm{LFn}}$ & $3.37 *$ & $3.10^{*}$ \\
$P_{\mathrm{LFrn}}$ & 1.76 & 1.35 \\
$P_{\mathrm{LFexn}}$ & 3.32 & 2.94 \\
\hline \hline
\end{tabular}

Table 2 shows percentage change between Relax (R) 
Table 2. Percentage change between Relax (R) and each stage for each HRV and PRV indices. Values in [\%] p-value $<0.05$

\begin{tabular}{lrrrrrrrrrr}
\hline \hline & \multicolumn{2}{c}{ PRB vs R } & \multicolumn{2}{c}{ PRS vs R } & \multicolumn{2}{c}{ SA vs R } & \multicolumn{2}{c}{ ST vs R } & \multicolumn{2}{c}{ V vs R } \\
\cline { 2 - 11 } & HRV & \multicolumn{1}{c}{ PRV } & \multicolumn{1}{c}{ HRV } & \multicolumn{1}{c}{ PRV } & HRV & \multicolumn{1}{c}{ PRV } & HRV & PRV & HRV & PRV \\
\hline$P_{\mathrm{HF}}$ & $10.39^{*}$ & $9.27^{*}$ & $21.69^{*}$ & $19.43^{*}$ & 3.34 & -8.39 & 13.49 & 0.22 & $14.20^{*}$ & -2.16 \\
$P_{\mathrm{HFr}}$ & $19.04^{*}$ & $16.34^{*}$ & $24.06^{*}$ & $20.24^{*}$ & -0.81 & $-14.24^{*}$ & 5.19 & -9.32 & 11.37 & -7.84 \\
$P_{\mathrm{HFex}}$ & $12.30^{*}$ & $11.13^{*}$ & $20.82^{*}$ & $18.55^{*}$ & 7.88 & -3.26 & $23.54^{*}$ & 13.39 & $19.88^{*}$ & 6.06 \\
$P_{\mathrm{HFn}}$ & 2.44 & 2.10 & $6.49^{*}$ & $5.86^{*}$ & -0.12 & -2.81 & $-8.05^{*}$ & $-10.96^{*}$ & $7.24^{*}$ & 3.99 \\
$P_{\text {HFrn }}$ & $4.73^{*}$ & $4.24^{*}$ & $6.84^{*}$ & $5.92^{*}$ & -1.57 & $-4.41^{*}$ & $-9.98^{*}$ & $-12.73^{*}$ & $5.55^{*}$ & 2.09 \\
$P_{\text {HFexn }}$ & $2.90^{*}$ & 2.53 & $6.38^{*}$ & $5.70^{*}$ & 1.22 & -1.39 & -4.70 & $-7.01^{*}$ & $9.14^{*}$ & $6.16^{*}$ \\
$L F / H F$ & -11.58 & -9.44 & $-31.13^{*}$ & $-26.88^{*}$ & -0.27 & 9.80 & $24.41^{*}$ & $31.64^{*}$ & $-41.59^{*}$ & $-23.17^{*}$ \\
$L F / H F r$ & $-23.50^{*}$ & $-18.66^{*}$ & $-35.46^{*}$ & $-28.33^{*}$ & 3.8 & $14.36^{*}$ & $31.12^{*}$ & $37.69^{*}$ & $-37.19^{*}$ & -16.75 \\
$L F / H F e x$ & $-14.03^{*}$ & -11.74 & $-29.71^{*}$ & $-25.51^{*}$ & -5.09 & 5.44 & 14.63 & $21.38^{*}$ & $-51.51^{*}$ & $-33.80^{*}$ \\
$P_{\mathrm{LF}}$ & 0.12 & 0.79 & -2.98 & -2.52 & 2.20 & 1.34 & $34.01^{*}$ & $31.17^{*}$ & $-22.46^{*}$ & $-26.79^{*}$ \\
$P_{\mathrm{LFn}}$ & -2.06 & -1.81 & $-5.63^{*}$ & $-5.08^{*}$ & 0.21 & 2.34 & $5.81^{*}$ & $7.80^{*}$ & $-6.13^{*}$ & -3.25 \\
$P_{\mathrm{LFrn}}$ & $-3.8^{*}$ & $-3.51^{*}$ & $-5.66^{*}$ & $-4.92^{*}$ & 1.38 & $3.48^{*}$ & $6.81^{*}$ & $8.58^{*}$ & $-4.15^{*}$ & -1.39 \\
$P_{\mathrm{LFexn}}$ & $-3.19^{*}$ & -2.86 & $-6.73^{*}$ & $-5.98^{*}$ & -0.90 & 1.72 & 4.28 & $6.32^{*}$ & $-9.88^{*}$ & $-6.51^{*}$ \\
\hline \hline
\end{tabular}

Table 3. Relative error between HRV and PRV indices, and mean Respiration frequency for each stage. Values in [\%] $*$ p-value $<0.05$

\begin{tabular}{lrrrrrc}
\hline \hline & PRB & \multicolumn{1}{c}{ PRS } & \multicolumn{1}{c}{$\mathrm{R}$} & \multicolumn{1}{c}{$\mathrm{SA}$} & \multicolumn{1}{c}{$\mathrm{ST}$} & $\mathrm{V}$ \\
\hline$P_{\mathrm{HF}}$ & $-24.56^{*}$ & $-22.58^{*}$ & $-26.11^{*}$ & $-12.47^{*}$ & -9.34 & -5.92 \\
$P_{\mathrm{HFr}}$ & $-28.61^{*}$ & $-26.49^{*}$ & $-32.91^{*}$ & $-17.29^{*}$ & -15.27 & -9.24 \\
$P_{\mathrm{HFex}}$ & $-21.07^{*}$ & $-19.26^{*}$ & $-22.68^{*}$ & -9.44 & -8.31 & -4.63 \\
$P_{\mathrm{HFn}}$ & $-4.54^{*}$ & $-4.20^{*}$ & $-4.90^{*}$ & -2.16 & -2.15 & -1.36 \\
$P_{\mathrm{HFrn}}$ & $-5.17^{*}$ & $-4.68^{*}$ & $-5.71^{*}$ & -2.84 & -3.13 & -1.97 \\
$P_{\mathrm{HF} \times x}$ & $-3.79^{*}$ & $-3.44^{*}$ & $-4.19^{*}$ & -1.50 & -1.95 & -0.88 \\
$L F / H F$ & $15.45^{*}$ & $14.30^{*}$ & $17.07^{*}$ & 7.81 & 8.30 & 4.65 \\
$L F / H F r$ & $18.10^{*}$ & $16.95^{*}$ & $21.31^{*}$ & 11.62 & 13.02 & 7.54 \\
$L F / H F e x$ & $13.00^{*}$ & $11.91^{*}$ & $14.75^{*}$ & 5.27 & 7.43 & 3.47 \\
$P_{\mathrm{LF}}$ & -5.29 & -5.06 & -4.58 & -3.67 & -0.26 & -1.00 \\
$P_{\mathrm{LFn}}$ & $3.52^{*}$ & $3.25^{*}$ & $3.75^{*}$ & 1.65 & 1.67 & 1.07 \\
$P_{\mathrm{LFm}}$ & $3.84^{*}$ & $3.43^{*}$ & $4.11^{*}$ & 2.02 & 2.25 & 1.50 \\
$P_{\mathrm{LFexn}}$ & $3.60^{*}$ & $3.22^{*}$ & $3.91^{*}$ & 1.35 & 1.81 & 0.86 \\
\hline$F_{\mathrm{R}}(k)$ & 0.26 & 0.26 & 0.27 & 0.30 & 0.34 & 0.33 \\
\hline \hline
\end{tabular}


and others stages for each HRV and PRV indices. Particularly for SA stage, only PRV indices related to HF band centered at $F_{\mathrm{R}}(k)$ are able to distinguish SA from R stage. Discrimination capability among PRB, PRS, ST stages and $\mathrm{R}$ are equivalent between HRV and PRV indices. Most of the HRV indices on $\mathrm{V}$ stage are significant different to HRV indices on R. In contrasts, just few PRV indices on V stage are significant different to PRV indices on R.

Relative error between HRV and PRV indices for each stage is shown in Table 3. Besides $P_{\mathrm{HF}}$ and $P_{\mathrm{HFr}}$, all HRV and PRV indices on SA, ST and V are similar. Only $P_{\mathrm{LF}}$, among others HRV and PRV indices, is not different on PRB, PRS and R stages.

\section{Discussion and conclusions}

Significant differences between PRB and PRS are not expected due to the experimental design and in order to use R stage as reference. However, they were observed for classic HF band indices and indices related to HF extended band and therefore, these indices should not be used to estimate stress levels. May be these indices are an overestimation of sympathetic activity and an underestimation of parasympathetic activity as is suggested in [5].

The PLF values from HRV and PRV are similar among analyzed stages, but only distinguish ST and V form R. Due to the fact that every time window where $F_{\mathrm{R}}(k)$ falls within the LF band were excluded, PLF could be interpreted as sympathetic activity. In this way, there is an increase of sympathetic activity on ST and a decrease of sympathetic activity on $\mathrm{V}$. According to rPHF there are no differences on parasympathetic activity during stress stages ST, SA and V.

Concerning about $\mathrm{HF}$ band centered at $F_{\mathrm{R}}(k)$, both HRV and PRV indices can distinguish between relax and prerelax stages (PRB and PRS). However, between R and V, PRV indices did not show significant differences while most of the HRV indices did. On the other hand, PRV indices showed significant differences between $\mathrm{R}$ and $\mathrm{SA}$ while none of HRV indices did.

Pulse arrival time (PAT) can be defined as the time that the pressure wave takes to propagate to the periphery from the time that a beat is detected at the ECG signal. PAT variability is the difference between HRV and PRV. During stress stages (ST, SA and V) there is not significant differences between HRV and PRV. In contrasts, most of the indices showed significant differences between PRV and HRV on relax stages (SA, ST, V). These results suggest that PAT variability is higher during relax, that could be explained by a low respiration frequency.

\section{Acknowledgements}

This work was funded by grant FIS-PI12/00514 of the MINECO, by FEDER under project TIN2014-53567-R, by Grupo Consolidado BSICoS ref:T96 from DGA and European Social Fund (EU) and by the Centro de Investigación Biomédica en Red sobre Bioingeniería, Biomateriales y Nanomedicina (CIBER-BBN) at the Instituto de Salud Carlos III de España.

\section{References}

[1] Charmandari E, Tsigos C, Chrousos G. Endocrinology Of The Stress Response. Annual Review of Physiology mar 2005;67(1):259-284. ISSN 0066-4278.

[2] Habib KE, Gold PW, Chrousos GP. Neuroendocrinology Of Stress. Endocrinology and Metabolism Clinics of North America sep 2001;30(3):695-728. ISSN 08898529.

[3] Gil E, Orini M, Bailón R, Vergara JM, Mainardi L, Laguna P. Photoplethysmography pulse rate variability as a surrogate measurement of heart rate variability during non-stationary conditions. Physiol Meas sep 2010;31(9):1271-1290. ISSN 0967-3334.

[4] Aguilo J, Ferrer-Salvans P, Garcia-Rozo A, Armario A, Corbi A, Cambra FJ, Bailen R, Gonzalez-Marcos A, Caja G, Aguilo S, Lopez-Anton R, Arza-Valdes A, Garzon-Rey JM. Project ES3: attempting to quantify and measure the level of stress. Revista de neurologia 2015;61(9):405-415.

[5] Hernando A, Lazaro J, Gil E, Arza Valdes A, Garzon-Rey JM, Lopez-Anton R, de la Camara C, Laguna P, Aguilo J, Bailon R. Inclusion of respiratory frequency information in heart rate variability analysis for stress assessment. IEEE journal of Journal of Biomedical and Health Informatics jul 2016;20(4):1-1. ISSN 2168-2194.

[6] Martínez JP, Almeida R, Olmos S, Rocha AP, Laguna P. A Wavelet-Based ECG Delineator: Evaluation on Standard Databases. IEEE transactions on biomedical engineering 2004;51(4):570-581.

[7] Mateo J, Laguna P. Analysis of heart rate variability in the presence of ectopic beats using the heart timing signal. IEEE transactions on biomedical engineering mar 2003; 50(3):334-43. ISSN 0018-9294.

[8] Gil E, Mendez M, Vergara JM, Cerutti S, Bianchi AM, Laguna P. Discrimination of Sleep-Apnea-Related Decreases in the Amplitude Fluctuations of PPG Signal in Children by HRV Analysis. IEEE transactions on biomedical engineering 2009;56(4):267-277.

[9] Lazaro J, Gil E, Vergara JM, Laguna P. Pulse Rate Variability Analysis for Discrimination of Sleep-Apnea-Related Decreases in the Amplitude Fluctuations of Pulse Photoplethysmographic Signal in Children. IEEE Journal of Biomedical and Health Informatics jan 2014;18(1):240-246. ISSN 21682194.

Address for correspondence:

Jorge Mario Garzón-Rey

Universitat Autònoma de Barcelona, spain

E-mail: jorgmario.garzon@uab.cat 